\title{
Solving smoking epidemic model of fractional order using a modified homotopy analysis transform method
}

\author{
P. Veeresha ${ }^{1}$ - D. G. Prakasha ${ }^{1} \cdot$ Haci Mehmet Baskonus ${ }^{2}$
}

Received: 30 November 2018 / Accepted: 10 April 2019 / Published online: 25 April 2019

(c) The Author(s) 2019

\begin{abstract}
The pivotal aim of the present work is to obtain an approximated analytical solution for the fractional smoking epidemic model with the aid of a novel technique called $q$-homotopy analysis transform method ( $q$-HATM). The considered nonlinear mathematical model has been effectively employed to elucidate the evolution of smoking in a population and its impact on public health in a community. We find some new approximate solutions in a series form, which converges rapidly, and the proposed algorithm provides auxiliary parameters, which are very reliable and feasible in controlling the convergence of obtained approximate solutions. Further, we present novel simulations for all cases of results to validate the applicability and effectiveness of proposed scheme. The outcomes of the study reveal that the $q$-HATM is computationally very effective to analyse nonlinear fractional differential equations arises in daily life problems.
\end{abstract}

Keywords Smoking model $\cdot q$-Homotopy analysis transform method $\cdot$ Caputo fractional derivative $\cdot$ Laplace transform . Epidemic model

\section{Introduction}

In 1766, Swiss mathematician and physicist Bernoulli [1] established and nurtured the idea of mathematical modelling for spread of disease, which gave birth to the start of modern epidemiology. Further, Ross [2] also presented the modelling of infectious disease in the beginning of twentieth century and explains the nature of epidemic models by using the law of mass action. Epidemic models have been extensively employed to study epidemiological processes which include transmission of contagious diseases. This kind of model has also been applied to study the dissemination of social habits, such as the alcohol consumption [3], obesity epidemics [4], cocaine consumption [5], smoking habit [6]

D. G. Prakasha

prakashadg@gmail.com; dgprakasha@kud.ac.in

P. Veeresha

viru0913@gmail.com

Haci Mehmet Baskonus

hmbaskonus@gmail.com

1 Department of Mathematics, Faculty of Science \& Technology, Karnatak University, Dharwad, India

2 Department of Mathematics, Faculty of Education, Harran University, Sanliurfa, Turkey and many more. Among these models, smoking model is one of the most attractive models for the last few decades for researchers. The World Health Organization proclaimed that smoking causes 250 million children and adolescent deaths and predicted that more than 10 million people will perish due to smoking-related diseases every year by 2030 [7]. The main effects of short-term smoking are the high blood pressure, stained teeth, bad breath and coughing. In recent years, lung cancer, throat cancer, mouth cancer, stomach ulcers, heart disease and gum disease are the main threatening due to long-term smoking. Therefore, use of smoking treated as a leading global public health problem. Smoking can also spread in similar with the spread of many infectious diseases, through social contact. Thus, mathematical modelling has been widely used to investigate the behaviour of smoking. In 2000, Castillo-Garsow et al. [8] first time suggested a simple mathematical model for giving up smoking. They address a scheme by dividing the population into three classes: smokers $(S)$, potential smokers $(P)$ and quit smokers $(Q)$. The local stability was proved by using the Routh-Hurwitz criterion while the global stability was only conjectured by using numerous simulations. In the recent years, many researches started to work on mathematical modelling of smoking phenomena [9-15] and these models 
give us an analytic framework in which to address specific reasons regarding smoking dynamics.

The concept of fractional calculus (FC) debated on 1695 and was firstly put forward by Guillaume de l'Hopital. FC recently showed a tremendous consideration and attention, which is the general expansion of the integer-order calculus to arbitrary order. Modelling by using the perception of FC penetrates the essential fundamentals for many dynamical systems. The biological process that modelled through arbitrary-order derivatives carries information about its present as well as past states [16, 17]. Derivatives and integrals of fractional order consider the system memory, hereditary properties and non-local distributed effects. These effects are essential for portraying the real-world problems $[18,19]$.

Mathematical models involving fractional differential equations have been proven valuable in understanding the dynamics of smoking. Many researchers developed the fractional-order model to study diffusion equation to predict the effect of smoking. Several powerful techniques for finding solutions for these models have been found in the literature [20-22]. In 1992, Liao [23, 24] proposed homotopy analysis method (HAM), and it has been effectively employing to find the solution for problems arises in science and technology. The HAM is based on construction of a homotopy which continuously deforms an initial guess approximation to the exact solution of the given problem. The $q$-HATM was proposed by Singh et al. [25], and which is an elegant amalgamation of $q$-HAM and Laplace transform.

The enhancement of proposed technique is its proficiency of amalgamating two strong algorithms to solve linear and nonlinear fractional differential equations both numerically as well analytically. Since, the techniques with perturbation, linearization or discretization we obtained only approximate solutions for nonlinear complex problems. These problems were appraised by exerting different schemes having their own limitations and weakness including; more time for evaluation, massive computational work and obtaining the divergent results. But, the proposed technique is free from any assumption, discretization and perturbation. The proposed algorithm has many sturdy properties including straightforward solution procedure and promising large convergence region. It is worth revealing that the Laplace transform with semi-analytical techniques requires less C.P.U time to evaluate solution for nonlinear complex models arised in science and technology. The $q$-HATM solution involves with two auxiliary parameters $\hbar$ and $n$, which helps us to adjust and control the convergence of the solution. We can say that the proposed technique can decrease the computation of the time and work as compared with other traditional techniques while maintaining the great efficiency of the obtained results. Due to these factors, recently, many authors are employed $q$-HATM to analyse the complex problems occur in science and engineering [26-29].
In present framework, we find the new approximate analytic solution for fractional smoking epidemic model. The proposed model permits incorporation of the memory effect involved in the fractional order, and this property is important in describing the biological problems. Moreover, $q$-HATM is applied to find the numerical solutions for the system of equations describing smoking model.

\section{Preliminaries}

In this section, we recall some definitions and properties of fractional calculus and Laplace transform

Definition 1 The fractional integral of a function $f(t) \in C_{\xi}(\xi \geq-1)$ and of order $\mu>0$, initially defined by Riemann-Liouville which is presented $[30,31]$ as

$$
J^{\mu} f(t)=\frac{1}{\Gamma(\mu)} \int_{0}^{t}(t-\vartheta)^{\mu-1} f(\vartheta) \mathrm{d} \vartheta,
$$

$J^{0} f(t)=f(t)$.

Definition 2 The fractional derivative of $f \in C_{-1}^{n}$ in the Caputo [32] sense is defined as

$D_{t}^{\mu} f(t)= \begin{cases}\frac{d^{n} f(t)}{\mathrm{d} t^{n}}, & \mu=n \in N, \\ \frac{1}{\Gamma(n-\mu)} \int_{0}^{t}(t-\vartheta)^{n-\mu-1} f^{(n)}(\vartheta) \mathrm{d} \vartheta, & n-1<\mu<n, n \in \mathbb{N} .\end{cases}$

Definition 3 The Laplace transform (LT) of a Caputo fractional derivative $D_{t}^{\mu} f(t)$ is represented [33] as

$\mathcal{L}\left[D_{t}^{\mu} f(t)\right]=s^{\mu} F(s)-\sum_{r=0}^{n-1} s^{\mu-r-1} f^{(r)}\left(0^{+}\right),(n-1<\mu \leq n)$,

where $F(s)$ symbolizes the Laplace transform of the function $f(t)$.

\section{Fractional mathematical model of smoking epidemic model in Caputo fractional derivatives}

It is an important and effective way to understand the biological problems by establishing mathematical models and analyzing their dynamical behaviours. In the present investigation, we consider the system of five nonlinear differential 
equations describing the smoking epidemic model. Let the total population size at time $t$ is represented by $N(t)$. We separate the population $N(t)$ into five subgroups, namely potential smoker $P(t)$, smoker $S(t)$, occasional smoker $O(t)$, permanently quit smoker $L(t)$ and temporarily quit smoker $Q(t)$. The proposed smoking model is presented as [34]:

$\frac{\partial P}{\partial t}=\lambda-\beta P S-\eta P$,

$\frac{\partial O}{\partial t}=\beta P S-\alpha_{1} O-\eta O$,

$\frac{\partial S}{\partial t}=\alpha_{1} O+\alpha_{2} S Q-(\eta+\gamma) S$,

$\frac{\partial Q}{\partial t}=-\alpha_{2} S Q-\eta Q+\gamma(1-\delta) S$

$\frac{\partial L}{\partial t}=\delta \gamma S-\eta L$

In the above system, $\lambda$ denotes the recruitment rate in the potential smoker, $\beta$ indicates the effective contact rate between potential smoker and smoker, $\eta$ symbolizes the natural death rate, $\gamma$ stands for rate of quitting smoking, $\delta$ represents the remaining fraction of smoking who permanently quit smoking, $\alpha_{1}$ is the rate at which occasional smokers becomes regular smoker, and $\alpha_{2}$ indicates the contact rate between smoker and temporary quitters who revert back to smoking. The parameters used in the system of Eqs. 4-8 are specified in Table 1.

Thus, in order to introduce the effect of non-locality, we moderate the above system by substituting the time derivative by the Caputo fractional derivative of the form:

$D_{t}^{\mu} P=\lambda-\beta P S-\eta P$,

$D_{t}^{\mu} O=\beta P S-\alpha_{1} O-\eta O$,

$D_{t}^{\mu} S=\alpha_{1} O+\alpha_{2} S Q-(\eta+\gamma) S, \quad 0<\mu \leq 1$

$D_{t}^{\mu} Q=-\alpha_{2} S Q-\eta Q+\gamma(1-\delta) S$

$D_{t}^{\mu} L=\delta \gamma S-\eta L$, with initial conditions

$P(0)=\epsilon_{1}, O(0)=\epsilon_{2}, S(0)=\epsilon_{3}, Q(0)=\epsilon_{4}, L(0)=\epsilon_{5}$.

\section{Equilibrium point and stability}

For equilibrium point [35], in system (9) we consider

$D_{t}^{\mu} P(t)=D_{t}^{\mu} O(t)=D_{t}^{\mu} S(t)=D_{t}^{\mu} Q(t)=D_{t}^{\mu} L(t)=0 ;$

we obtained disease-free equilibria as

$E_{0}=(P, 0, S, 0,0)$,

and endemic equilibria of the system is

$E^{*}=\left(P^{*}, O^{*} S^{*}, Q^{*}, L^{*}\right)$,

where

$P^{*}=\frac{\lambda}{\beta+\eta}, O^{*}=\frac{\beta \lambda S}{(\beta S+\eta)\left(\alpha_{1}+\eta\right)}, Q^{*}=\frac{\gamma(1-\delta) S}{\alpha_{2} S+\eta}, L^{*}=\frac{\delta \gamma S}{\eta}$.

Theorem 1 [35] The disease-free equilibrium $E^{*}$ is locally asymptotically stable if $R_{0}<1$, otherwise unstable.

\section{Fundamental idea of $q$-homotopy analysis transform method}

To present the fundamental idea of proposed method [36, 37], consider the nonlinear non-homogeneous partial differential equation of fractional order:

$D_{t}^{\mu} v(x, t)+\mathcal{R} v(x, t)+\mathcal{N} v(x, t)=f(x, t), \quad n-1<\mu \leq n$,

where $D_{t}^{\mu} v(x, t)$ represents the Caputo fractional derivative of the function $v(x, t), \mathcal{R}$ and $\mathcal{N}$, respectively, specifies the linear and nonlinear differential operator, and $f(x, t)$ represents the source term. Now, by employing the $L T$ on Eq. (13), we get

$$
\begin{aligned}
& s^{\mu} \mathcal{L}[v(x, t)]-\sum_{k=0}^{n-1} s^{\mu-k-1} v^{(k)}(x, 0) \\
& \quad+\mathcal{L}[\mathcal{R} v(x, t)]+\mathcal{L}[\mathcal{N} v(x, t)]=\mathcal{L}[f(x, t)] .
\end{aligned}
$$

Table 1 Description of parameters used in system (9) and their specific values

\begin{tabular}{lll}
\hline Parameters & Descriptions & $\begin{array}{l}\text { Values (unit: 1/ } \\
\text { time) }\end{array}$ \\
\hline$\lambda$ & Recruitment rate in $P$ & 1 \\
$\beta$ & Effective contact rate between $S$ and $P$ & 0.14 \\
$\eta$ & Natural death rate & 0.05 \\
$\alpha_{1}$ & Rate at which occasional smokers become regular smokers & 0.002 \\
$\alpha_{2}$ & Contact rate between smokers and temporary quitters who revert back to & 0.0025 \\
$\gamma$ & smoking & 0.8 \\
$\delta$ & Rate of quitting smoking & 0.1 \\
\hline
\end{tabular}


On simplifying Eq. (14), we have

$$
\begin{aligned}
& \mathcal{L}[v(x, t)]-\frac{1}{s^{\mu}} \sum_{k=0}^{n-1} s^{\mu-k-1} v^{k}(x, 0) \\
& +\frac{1}{s^{\mu}}\{\mathcal{L}[\mathcal{R} v(x, t)]+\mathcal{L}[\mathcal{N} v(x, t)]-\mathcal{L}[f(x, t)]\}=0 .
\end{aligned}
$$

According to homotopy analysis method [24], the nonlinear operator defined as

$$
\begin{aligned}
\mathcal{N}[\varphi(x, t ; q)]= & \mathcal{L}[\varphi(x, t ; q)]-\frac{1}{s^{\mu}} \sum_{k=0}^{n-1} s^{\mu-k-1} \varphi^{(k)}(x, t ; q)\left(0^{+}\right) \\
& +\frac{1}{s^{\mu}}\{\mathcal{L}[\mathcal{R} \varphi(x, t ; q)]+L[\mathcal{N} \varphi(x, t ; q)]-L[f(x, t)]\},
\end{aligned}
$$

where $q \in\left[0, \frac{1}{n}\right]$, and $\varphi(x, t ; q)$ is a real function of $x, t$ and $q$.

We construct a homotopy for nonzero auxiliary function as follows:

$(1-n q) \mathcal{L}\left[\varphi(x, t ; q)-v_{0}(x, t)\right]=\hbar q \mathcal{N}[\varphi(x, t ; q)]$,

where $\mathcal{L}$ be a symbol of the $L T, \hbar \neq 0$ is an auxiliary parameter, $q \in\left[0, \frac{1}{n}\right](n \geq 1)$ is the embedding parameter, $v_{0}(x, t)$ is an initial guess of $v(x, t)$ and $\varphi(x, t ; q)$ is an unknown function. The following results hold for $q=0$ and $q=\frac{1}{n}$ :

$\varphi(x, t ; 0)=v_{0}(x, t), \quad \varphi\left(x, t ; \frac{1}{n}\right)=v(x, t)$,

respectively. Thus, by amplifying $q$ from 0 to $\frac{1}{n}$, the solution $\varphi(x, t ; q)$ converges from $v_{0}(x, t)$ to the solution $v(x, t)$. Expanding the function $\varphi(x, t ; q)$ in series form by employing Taylor theorem near to $q$, one can get

$\varphi(x, t ; q)=v_{0}(x, t)+\sum_{m=1}^{\infty} v_{m}(x, t) q^{m}$

where

$v_{m}(x, t)=\left.\frac{1}{m !} \frac{\partial^{m} \varphi(x, t ; q)}{\partial q^{m}}\right|_{q=0}$.

On choosing the auxiliary linear operator, the initial guess is $v_{0}(x, t), n$, and $\hbar$, and the series (19) converges at $q=\frac{1}{n}$; then, it gives one of the solutions of the original nonlinear equation of the form

$v(x, t)=v_{0}(x, t)+\sum_{m=1}^{\infty} v_{m}(x, t)\left(\frac{1}{n}\right)^{m}$.

Now, differentiating Eq. (17) $m$ times with respect to $q$ and then dividing by $m$ ! and lastly taking $q=0$, which yields

$\mathcal{L}\left[v_{m}(x, t)-\mathrm{k}_{m} v_{m-1}(x, t)\right]=\hbar \Re_{m}\left(\vec{v}_{m-1}\right)$,

where the vectors are defined as $\vec{v}_{m}=\left\{v_{0}(x, t), v_{1}(x, t), \ldots, v_{m}(x, t)\right\}$.

Applying the inverse $L T$ on Eq. (22), it provides the following recursive equation

$v_{m}(x, t)=\mathrm{k}_{m} v_{m-1}(x, t)+\hbar \mathcal{L}^{-1}\left[\Re_{m}\left(\vec{v}_{m-1}\right)\right]$,

where

$$
\begin{aligned}
\mathfrak{R}_{m}\left(\vec{v}_{m-1}\right)= & L\left[v_{m-1}(x, t)\right]-\left(1-\frac{\mathrm{k}_{m}}{n}\right) \\
& \times\left(\sum_{k=0}^{n-1} s^{\mu-k-1} v^{(k)}(x, 0)+\frac{1}{s^{\mu}} L[f(x, t)]\right) \\
& +\frac{1}{s^{\mu}} L\left[R v_{m-1}+\mathcal{H}_{m-1}\right]
\end{aligned}
$$

and

$\mathrm{k}_{m}= \begin{cases}0, & m \leq 1, \\ n, & m>1 .\end{cases}$

In Eq. (25), $\mathcal{H}_{m}$ denotes homotopy polynomial and defined as

$\mathcal{H}_{m}=\frac{1}{m !}\left[\frac{\partial^{m} \varphi(x, t ; q)}{\partial q^{m}}\right]_{q=0} \quad$ and $\quad \varphi(x, t ; q)=\varphi_{0}+q \varphi_{1}+q^{2} \varphi_{2}+\cdots$.

By Eqs. (24) and (25), we have

$$
\begin{aligned}
v_{m}(x, t)= & \left(\mathrm{k}_{m}+\hbar\right) v_{m-1}(x, t)-\left(1-\frac{\mathrm{k}_{m}}{n}\right) \mathcal{L}^{-1}\left(\sum_{k=0}^{n-1} s^{\mu-k-1} v^{(k)}(x, 0)\right. \\
& \left.+\frac{1}{s^{\mu}} \mathcal{L}[f(x, t)]\right)+\hbar \mathcal{L}^{-1}\left\{\frac{1}{s^{\mu}} L\left[R v_{m-1}+\mathcal{H}_{m-1}\right]\right\} .
\end{aligned}
$$

Finally, on solving Eq. (28), we get the iterative terms of $v_{m}(x, t)$. The $q$-HATM series solution is presented by

$v(x, t)=\sum_{m=0}^{\infty} v_{m}(x, t)$.

\section{Convergence analysis of $q$-HATM solution}

Theorem 2 (Uniqueness theorem) [38] The solution for the nonlinear fractional differential Eq. (13) obtained by $q$-HATM is unique for every $\beta \in(0,1)$, where $\beta=(n+\hbar)+\hbar(\epsilon+\mu) T$.

Theorem 3 (Convergence theorem) [38] Let X be a Banach space and $F: X \rightarrow X$ be a nonlinear mapping. Assume that $\|F(v)-F(w)\| \leq \beta\|v-w\|, \quad \forall a, b \in X$,

then $F$ has a fixed point in view of Banach fixed point theory [39]. Moreover, for the arbitrary selection of $a_{0}, b_{0} \in X$, the sequence generated by the q-HATM converges to fixed point of $F$ and 
$\left\|v_{m}-v_{n}\right\| \leq \frac{\beta^{n}}{1-\beta}\left\|v_{1}-v_{0}\right\|, \quad \forall a, b \in X$.

\section{q-HATM solution for system of time-fractional smoking epidemic effect equations}

In this section, we illustrate some numerical solutions of the model (9) for different values of the parameters, and we show that these solutions are in agreement with the qualitative behaviour of the solutions. Consider time-fractional smoking epidemic effect equations

$$
\begin{aligned}
\frac{d^{\mu} P}{\mathrm{~d} t^{\mu}} & =\lambda-\beta P S-\eta P, \\
\frac{d^{\mu} O}{\mathrm{~d} t^{\mu}} & =\beta P S-\alpha_{1} O-\eta O, \\
\frac{d^{\mu} S}{\mathrm{~d} t^{\mu}} & =\alpha_{1} O+\alpha_{2} S Q-(\eta+\gamma) S, \quad 0<\mu \leq 1, \\
\frac{d^{\mu} Q}{\mathrm{~d} t^{\mu}} & =-\alpha_{2} S Q-\eta Q+\gamma(1-\delta) S, \\
\frac{d^{\mu} L}{\mathrm{~d} t^{\mu}} & =\delta \gamma S-\eta L,
\end{aligned}
$$

with initial conditions

$$
P(0)=\epsilon_{1}, O(0)=\epsilon_{2}, S(0)=\epsilon_{3}, Q(0)=\epsilon_{4}, L(0)=\epsilon_{5} .
$$

Now, by performing $L T$ on system of Eqs. (31) and using conditions cited in Eq. (32), we have

$$
\begin{aligned}
& \mathcal{L}[P(t)]-\frac{1}{s}\left(\epsilon_{1}\right)-\frac{1}{s^{\mu}} \mathcal{L}\{\lambda-\beta P S-\eta P\}=0, \\
& \mathcal{L}[O(t)]-\frac{1}{s}\left(\epsilon_{2}\right)-\frac{1}{s^{\mu}} \mathcal{L}\left\{\beta P S-\alpha_{1} O-\eta O\right\}, \\
& \mathcal{L}[S(t)]-\frac{1}{s}\left(\epsilon_{3}\right)-\frac{1}{s^{\mu}} \mathcal{L}\left\{\alpha_{1} O+\alpha_{2} S Q-(\eta+\gamma) S\right\}, \\
& \mathcal{L}[Q(t)]-\frac{1}{S}\left(\epsilon_{4}\right)-\frac{1}{s^{\mu}} \mathcal{L}\left\{-\alpha_{2} S Q-\eta Q+\gamma(1-\delta) S\right\}, \\
& \mathcal{L}[L(t)]-\frac{1}{S}\left(\epsilon_{5}\right)-\frac{1}{s^{\mu}} \mathcal{L}\{\delta \gamma S-\eta L\} .
\end{aligned}
$$

Define the nonlinear operator as

$$
\begin{aligned}
N^{1} & {\left[\varphi_{1}(t ; q), \varphi_{2}(t ; q), \varphi_{3}(t ; q), \varphi_{4}(t ; q), \varphi_{5}(t ; q)\right] } \\
& =\mathcal{L}\left[\varphi_{1}(t ; q)\right]-\frac{1}{s}\left(\epsilon_{1}\right)-\frac{1}{s^{\mu}} \mathcal{L}\left\{\lambda-\beta \varphi_{1}(t ; q) \varphi_{3}(t ; q)-\eta \varphi_{1}(t ; q)\right\}, \\
N^{2} & {\left[\varphi_{1}(t ; q), \varphi_{2}(t ; q), \varphi_{3}(t ; q), \varphi_{4}(t ; q), \varphi_{5}(t ; q)\right] } \\
& =\mathcal{L}\left[\varphi_{2}(t ; q)\right]-\frac{1}{s}\left(\epsilon_{2}\right)-\frac{1}{s^{\mu}} \mathcal{L}\left\{\beta \varphi_{1}(t ; q) \varphi_{3}(t ; q)-\alpha_{1} \varphi_{2}(t ; q)-\eta \varphi_{2}(t ; q)\right\}, \\
N^{3} & {\left[\varphi_{1}(t ; q), \varphi_{2}(t ; q), \varphi_{3}(t ; q), \varphi_{4}(t ; q), \varphi_{5}(t ; q)\right] } \\
& =\mathcal{L}\left[\varphi_{3}(t ; q)\right]-\frac{1}{s}\left(\epsilon_{3}\right)-\frac{1}{s^{\mu}} \mathcal{L}\left\{\alpha_{1} \varphi_{2}(t ; q)+\alpha_{2} \varphi_{3}(t ; q) \varphi_{4}(t ; q)-(\eta+\gamma) \varphi_{3}(t ; q)\right\}, \\
N^{4} & {\left[\varphi_{1}(t ; q), \varphi_{2}(t ; q), \varphi_{3}(t ; q), \varphi_{4}(t ; q), \varphi_{5}(t ; q)\right] } \\
& =\mathcal{L}\left[\varphi_{4}(t ; q)\right]-\frac{1}{s}\left(\epsilon_{4}\right)-\frac{1}{s^{\mu}} \mathcal{L}\left\{-\alpha_{2} \varphi_{3}(t ; q) \varphi_{4}(t ; q)-\eta \varphi_{4}(t ; q)+\gamma(1-\delta) \varphi_{3}(t ; q)\right\}, \\
N^{5} & {\left[\varphi_{1}(t ; q), \varphi_{2}(t ; q), \varphi_{3}(t ; q), \varphi_{4}(t ; q), \varphi_{5}(t ; q)\right] } \\
& =\mathcal{L}\left[\varphi_{5}(t ; q)\right]-\frac{1}{s}\left(\epsilon_{5}\right)-\frac{1}{s^{\mu}} \mathcal{L}\left\{\delta \gamma \varphi_{3}(t ; q)-\eta \varphi_{5}(t ; q)\right\} .
\end{aligned}
$$


By applying suggested algorithm, the deformation equation of $m$ th order is given as

$$
\begin{array}{r}
\mathcal{L}\left[P_{m}(x, t)-\mathrm{k}_{m} P_{m-1}(x, t)\right]=\hbar \mathcal{L}^{-1}\left\{\mathfrak{R}_{1, m}\left[\vec{P}_{m-1}, \vec{O}_{m-1}, \vec{S}_{m-1}, \vec{Q}_{m-1}, \vec{L}_{m-1}\right]\right\}, \\
\mathcal{L}\left[O_{m}(x, t)-\mathrm{k}_{m} O_{m-1}(x, t)\right]=\hbar \mathcal{L}^{-1}\left\{\mathfrak{R}_{2, m}\left[\vec{P}_{m-1}, \vec{O}_{m-1}, \vec{S}_{m-1}, \vec{Q}_{m-1}, \vec{L}_{m-1}\right]\right\}, \\
\mathcal{L}\left[S_{m}(x, t)-\mathrm{k}_{m} S_{m-1}(x, t)\right]=\hbar \mathcal{L}^{-1}\left\{\mathfrak{R}_{3, m}\left[\vec{P}_{m-1}, \vec{O}_{m-1}, \vec{S}_{m-1}, \vec{Q}_{m-1}, \vec{L}_{m-1}\right]\right\}, \\
\mathcal{L}\left[Q_{m}(x, t)-\mathrm{k}_{m} Q_{m-1}(x, t)\right]=\hbar \mathcal{L}^{-1}\left\{\mathfrak{R}_{4, m}\left[\vec{P}_{m-1}, \vec{O}_{m-1}, \vec{S}_{m-1}, \vec{Q}_{m-1}, \vec{L}_{m-1}\right]\right\}, \\
\mathcal{L}\left[L_{m}(x, t)-\mathrm{k}_{m} L_{m-1}(x, t)\right]=\hbar \mathcal{L}^{-1}\left\{\mathfrak{R}_{5, m}\left[\vec{P}_{m-1}, \vec{O}_{m-1}, \vec{S}_{m-1}, \vec{Q}_{m-1}, \vec{L}_{m-1}\right]\right\},
\end{array}
$$

where

$$
\begin{aligned}
& \mathfrak{R}_{1, m}\left[\vec{P}_{m-1}, \vec{O}_{m-1}, \vec{S}_{m-1}, \vec{Q}_{m-1}, \vec{L}_{m-1}\right] \\
& =\mathcal{L}\left[P_{m-1}(x, t)\right]-\left(1-\frac{k_{m}}{n}\right) \frac{1}{s}\left(\epsilon_{1}\right)-\frac{1}{s^{\mu}} \mathcal{L}\left\{\lambda-\beta \sum_{i=0}^{m-1} P_{i} S_{m-1-i}-\eta P_{m-1}\right\} \\
& \mathfrak{R}_{2, m}\left[\vec{P}_{m-1}, \vec{O}_{m-1}, \vec{S}_{m-1}, \vec{Q}_{m-1}, \vec{L}_{m-1}\right] \\
& =\mathcal{L}\left[O_{m-1}(x, t)\right]-\left(1-\frac{k_{m}}{n}\right) \frac{1}{s}\left(\epsilon_{2}\right)-\frac{1}{s^{\mu}} \mathcal{L}\left\{\beta \sum_{i=0}^{m-1} P_{i} S_{m-1-i}-\alpha_{1} O_{m-1}-\eta O_{m-1}\right\}, \\
& \mathfrak{R}_{3, m}\left[\vec{P}_{m-1}, \vec{O}_{m-1}, \vec{S}_{m-1}, \vec{Q}_{m-1}, \vec{L}_{m-1}\right] \\
& =\mathcal{L}\left[S_{m-1}(x, t)\right]-\left(1-\frac{k_{m}}{n}\right) \frac{1}{s}\left(\epsilon_{3}\right)-\frac{1}{s^{\mu}} \mathcal{L}\left\{\alpha_{1} O_{m-1}+\alpha_{2} \sum_{i=0}^{m-1} S_{i} Q_{m-1-i}-(\eta+\gamma) S_{m-1}\right\} \\
& \mathfrak{R}_{4, m}\left[\vec{P}_{m-1}, \vec{O}_{m-1}, \vec{S}_{m-1}, \vec{Q}_{m-1}, \vec{L}_{m-1}\right] \\
& \quad=\mathcal{L}\left[Q_{m-1}(x, t)\right]-\left(1-\frac{k_{m}}{n}\right) \frac{1}{s}\left(\epsilon_{4}\right)-\frac{1}{s^{\mu}} \mathcal{L}\left\{-\alpha_{2} \sum_{i=0}^{m-1} S_{i} Q_{m-1-i}-\eta Q_{m-1}+\gamma(1-\delta) S_{m-1}\right\} \\
& \mathfrak{R}_{5, m}\left[\vec{P}_{m-1}, \vec{O}_{m-1}, \vec{S}_{m-1}, \vec{Q}_{m-1}, \vec{L}_{m-1}\right] \\
& =\mathcal{L}\left[L_{m-1}(x, t)\right]-\left(1-\frac{k_{m}}{n}\right) \frac{1}{s}\left(\epsilon_{5}\right)-\frac{1}{s^{\mu}} \mathcal{L}\left\{\delta \gamma S_{m-1}-\eta L_{m-1}\right\} .
\end{aligned}
$$

By applying inverse Laplace transform on system of

Eq. (35), we get

$$
\begin{aligned}
& P_{m}(x, t)=\mathrm{k}_{m} P_{m-1}(x, t)+\hbar \mathcal{L}^{-1}\left\{\mathfrak{R}_{1, m}\left[\vec{P}_{m-1}, \vec{O}_{m-1}, \vec{S}_{m-1}, \vec{Q}_{m-1}, \vec{L}_{m-1}\right]\right\}, \\
& O_{m}(x, t)=\mathrm{k}_{m} O_{m-1}(x, t)+\hbar \mathcal{L}^{-1}\left\{\mathfrak{R}_{2, m}\left[\vec{P}_{m-1}, \vec{O}_{m-1}, \vec{S}_{m-1}, \vec{Q}_{m-1}, \vec{L}_{m-1}\right]\right\}, \\
& S_{m}(x, t)=\mathrm{k}_{m} S_{m-1}(x, t)+\hbar \mathcal{L}^{-1}\left\{\mathfrak{R}_{3, m}\left[\vec{P}_{m-1}, \vec{O}_{m-1}, \vec{S}_{m-1}, \vec{Q}_{m-1}, \vec{L}_{m-1}\right]\right\}, \\
& Q_{m}(x, t)=\mathrm{k}_{m} Q_{m-1}(x, t)+\hbar \mathcal{L}^{-1}\left\{\mathfrak{R}_{4, m}\left[\vec{P}_{m-1}, \vec{O}_{m-1}, \vec{S}_{m-1}, \vec{Q}_{m-1}, \vec{L}_{m-1}\right]\right\}, \\
& L_{m}(x, t)=\mathrm{k}_{m} L_{m-1}(x, t)+\hbar \mathcal{L}^{-1}\left\{\mathfrak{R}_{5, m}\left[\vec{P}_{m-1}, \vec{O}_{m-1}, \vec{S}_{m-1}, \vec{Q}_{m-1}, \vec{L}_{m-1}\right]\right\}
\end{aligned}
$$


On solving above system of equations by using initial conditions $P(0)=\epsilon_{1}=40, O(0)=\epsilon_{2}=10$, $S(0)=\epsilon_{3}=20, Q(0)=\epsilon_{4}=10, L(0)=\epsilon_{5}=5$, we have $S_{1}(t)=\frac{\hbar\left(-10 \alpha_{1}+20\left(\gamma+\eta-10 \alpha_{2}\right)\right) t^{\mu}}{\Gamma[\mu+1]}$ $P_{0}(t)=40, O_{0}(t)=10, S_{0}(t)=20, Q_{0}(t)=10, L_{0}(t)=5$,

$P_{1}(t)=\frac{\hbar(800 \beta+40 \eta-\lambda) t^{\mu}}{\Gamma[\mu+1]}$, $Q_{1}(t)=\frac{\hbar\left(10\left(2 \gamma(-1+\delta)+\eta+20 \alpha_{2}\right)\right) t^{\mu}}{\Gamma[\mu+1]}$,

$O_{1}(t)=\frac{\hbar\left(10\left(-80 \beta+\eta+\alpha_{1}\right)\right) t^{\mu}}{\Gamma[\mu+1]}$, $L_{1}(t)=\frac{\hbar(-20 \gamma \delta+5 \eta) t^{\mu}}{\Gamma[\mu+1]}$

$P_{2}(t)=\frac{(n+\hbar) \hbar(800 \beta+40 \eta-\lambda) t^{\mu}}{\Gamma[\mu+1]}$

$+\frac{\hbar^{2}\left(-\lambda+\eta(800 \beta+40 \eta-\lambda)+20 \beta\left(800 \beta+40 \gamma+80 \eta-\lambda-20 \alpha_{1}-400 \alpha_{2}\right)\right) t^{2 \mu}}{\Gamma[2 \mu+1]}$

$O_{2}(t)=\frac{(n+\hbar) \hbar\left(10\left(-80 \beta+\eta+\alpha_{1}\right)\right) t^{\mu}}{\Gamma[\mu+1]}$

$+\frac{10 \hbar^{2}\left(-1600 \beta^{2}-80 \beta \gamma-240 \beta \eta+\eta^{2}+2 \beta \lambda+(-40 \beta+2 \eta) \alpha_{1}+\alpha_{1}^{2}+800 \beta \alpha_{2}\right) t^{2 \mu}}{\Gamma[2 \mu+1]}$,

$$
\begin{aligned}
S_{2}(t)= & \frac{(n+\hbar) \hbar\left(-10 \alpha_{1}+20\left(\gamma+\eta-10 \alpha_{2}\right)\right) t^{\mu}}{\Gamma[\mu+1]} \\
& -\frac{10 \hbar^{2}\left(\alpha_{1}^{2}+\alpha_{1}\left(-80 \beta+\gamma+2 \eta-10 \alpha_{2}\right)-2\left((\gamma+\eta)^{2}-10(2 \gamma \delta+3 \eta) \alpha_{2}-100 \alpha_{2}^{2}\right)\right) t^{2 \mu}}{\Gamma[2 \mu+1]},
\end{aligned}
$$

$Q_{2}(t)=\frac{(n+\hbar) \hbar\left(10\left(2 \gamma(-1+\delta)+\eta+20 \alpha_{2}\right)\right) t^{\mu}}{\Gamma[\mu+1]}$

$+\frac{10 \hbar^{2}\left(-2 \gamma^{2}+2 \gamma^{2} \delta-4 \gamma \eta+4 \gamma \delta \eta+\eta^{2}+\alpha_{1}\left(\gamma-\gamma \delta-10 \alpha_{2}\right)+20(\gamma \delta+3 \eta) \alpha_{2}+200 \alpha_{2}^{2}\right) t^{2 \mu}}{\Gamma[2 \mu+1]}$,

$L_{2}(t)=\frac{(n+\hbar) \hbar(-20 \gamma \delta+5 \eta) t^{\mu}}{\Gamma[\mu+1]}+\frac{5 \hbar^{2}\left(-4 \gamma^{2} \delta-8 \gamma \delta \eta+\eta^{2}+2 \gamma \delta \alpha_{1}+40 \gamma \delta \alpha_{2}\right) t^{2 \mu}}{\Gamma[2 \mu+1]}$

$P_{3}(t)=\frac{(n+\hbar)^{2} \hbar(800 \beta+40 \eta-\lambda) t^{\mu}}{\Gamma[\mu+1]}$

$+\frac{(n+\hbar) \hbar^{2}\left(-\lambda+\eta(800 \beta+40 \eta-\lambda)+20 \beta\left(800 \beta+40 \gamma+80 \eta-\lambda-20 \alpha_{1}-400 \alpha_{2}\right)\right) t^{2 \mu}}{\Gamma[2 \mu+1]}$

$+\frac{\hbar^{3} t^{3 \mu}}{\Gamma[\mu+1]^{2} \Gamma[3 \mu+1]}\left(\left(10 t^{3 \mu} \hbar^{3} \beta(800 \beta+40 \eta-\lambda)\left(-\alpha_{1}+2\left(\gamma+\eta-10 \alpha_{2}\right)\right)\right) \Gamma[2 \mu+1]\right.$

$+\Gamma[\mu+1]^{2}\left(-\lambda+40\left(8000 \beta^{3}+\eta^{3}+400 \beta^{2}(\gamma+4 \eta)+20 \beta\left(\gamma^{2}+3 \gamma \eta+5 \eta^{2}\right)\right)\right.$

$-(20 \beta+\eta)(1+20 \beta+\eta) \lambda+400 \beta\left(-\alpha_{1}\left(-60 \beta+\gamma+3 \eta+\alpha_{1}\right)\right.$

$\left.\left.\left.+10\left(-4(10 \beta+\gamma \delta+2 \eta)+\alpha_{1}\right) \alpha_{2}-200 \alpha_{2}^{2}\right)\right)\right)$, 
Table 2 Numerical simulation using $q$-HATM for $P(t)$ at $n=1, \hbar=-1$ and using Table 1 for different $\mu$ with diverse values $t$

\begin{tabular}{llll}
\hline$P(t)$ & $\mu=0.8$ & $\mu=0.9$ & $\mu=1$ \\
\hline$t=0$ & 40 & 40 & 40 \\
$t=0.1$ & 28.0338 & 29.1162 & 30.7667 \\
$t=0.2$ & 28.5365 & 26.0052 & 25.6668 \\
$t=0.3$ & 35.8119 & 28.4733 & 24.7002 \\
$t=0.4$ & 48.4484 & 35.8752 & 27.8670 \\
$t=0.5$ & 65.6923 & 47.8402 & 35.1672 \\
$t=0.6$ & 87.0555 & 64.1152 & 46.6008 \\
$t=0.7$ & 112.189 & 84.5111 & 62.1678 \\
$t=0.8$ & 140.825 & 108.878 & 81.8682 \\
$t=0.9$ & 172.753 & 137.095 & 105.702 \\
$t=1.0$ & 207.799 & 169.057 & 133.669 \\
\hline
\end{tabular}

Table 3 Numerical study by employing $q$-HATM for $O(t)$ at $n=1, \hbar=-1$ and using Table 1 for different $\mu$ with distinct $t$

\begin{tabular}{llll}
\hline$O(t)$ & $\mu=0.8$ & $\mu=0.9$ & $\mu=1$ \\
\hline$t=0$ & 10 & 10 & 10 \\
$t=0.05$ & 18.4931 & 16.6946 & 15.0559 \\
$t=0.10$ & 21.6874 & 20.6741 & 19.0756 \\
$t=0.15$ & 22.3060 & 22.8892 & 22.0590 \\
$t=0.20$ & 20.9520 & 23.5858 & 24.0063 \\
$t=0.25$ & 17.9345 & 22.8977 & 24.9173 \\
$t=0.30$ & 13.4483 & 20.9135 & 24.7922 \\
$t=0.35$ & 7.63003 & 17.6978 & 23.6308 \\
$t=0.40$ & 3.58228 & 13.3004 & 21.4332 \\
$t=0.45$ & 1.61433 & 7.76208 & 18.1994 \\
$t=0.50$ & 0.58228 & 1.11636 & 13.9294 \\
\hline
\end{tabular}

Table 4 Numerical simulation using $q$-HATM for $S(t)$ at $n=1, \hbar=-1$ and using Table 1 for different $\mu$ with diverse $t$

\begin{tabular}{llll}
\hline$S(t)$ & $\mu=0.8$ & $\mu=0.9$ & $\mu=1$ \\
\hline$t=0$ & 20 & 20 & 20 \\
$t=0.1$ & 17.4502 & 17.9798 & 18.4244 \\
$t=0.2$ & 15.8891 & 16.4515 & 16.9938 \\
$t=0.3$ & 14.7230 & 15.1913 & 15.7080 \\
$t=0.4$ & 13.8383 & 14.1491 & 14.5671 \\
$t=0.5$ & 13.1806 & 13.2994 & 13.5711 \\
$t=0.6$ & 12.7172 & 12.6260 & 12.7201 \\
$t=0.7$ & 12.4259 & 12.1178 & 12.0138 \\
$t=0.8$ & 12.2905 & 11.7662 & 11.4525 \\
$t=0.9$ & 12.2987 & 11.5645 & 11.0360 \\
$t=1.0$ & 12.4407 & 11.5073 & 10.7645 \\
\hline & & &
\end{tabular}

Table 5 Numerical study by using $q$-HATM for $Q(t)$ at $n=1, \hbar=-1$ and using Table 1 for different $\mu$ with different $t$

\begin{tabular}{llll}
\hline$Q(t)$ & $\mu=0.8$ & $\mu=0.9$ & $\mu=1$ \\
\hline$t=0$ & 10 & 10 & 10 \\
$t=0.1$ & 12.0554 & 11.6331 & 11.2760 \\
$t=0.2$ & 13.2887 & 12.8520 & 12.4241 \\
$t=0.3$ & 14.1876 & 13.8408 & 13.4443 \\
$t=0.4$ & 14.8466 & 14.6413 & 14.3365 \\
$t=0.5$ & 15.3112 & 15.2749 & 15.1008 \\
$t=0.6$ & 15.6089 & 15.7550 & 15.7372 \\
$t=0.7$ & 15.7583 & 16.0913 & 16.2456 \\
$t=0.8$ & 15.7730 & 16.2908 & 16.6260 \\
$t=0.9$ & 15.6636 & 16.3594 & 16.8786 \\
$t=1.0$ & 15.4383 & 16.3015 & 17.0032 \\
\hline
\end{tabular}

Table 6 Numerical simulation using $q$-HATM for $L(t)$ at $n=1, \hbar=-1$ and using Table 1 for different $\mu$ with diverse $t$

\begin{tabular}{llll}
\hline$L(t)$ & $\mu=0.8$ & $\mu=0.9$ & $\mu=1$ \\
\hline$t=0$ & 5 & 5 & 5 \\
$t=0.1$ & 5.20537 & 5.16361 & 5.12807 \\
$t=0.2$ & 5.32615 & 5.28413 & 5.24228 \\
$t=0.3$ & 5.41201 & 5.38032 & 5.34263 \\
$t=0.4$ & 5.47262 & 5.45648 & 5.42913 \\
$t=0.5$ & 5.51271 & 5.51481 & 5.50176 \\
$t=0.6$ & 5.53511 & 5.55673 & 5.56054 \\
$t=0.7$ & 5.54178 & 5.58323 & 5.60545 \\
$t=0.8$ & 5.53414 & 5.59506 & 5.63651 \\
$t=0.9$ & 5.51326 & 5.59282 & 5.65371 \\
$t=1.0$ & 5.48004 & 5.57701 & 5.65705 \\
\hline
\end{tabular}

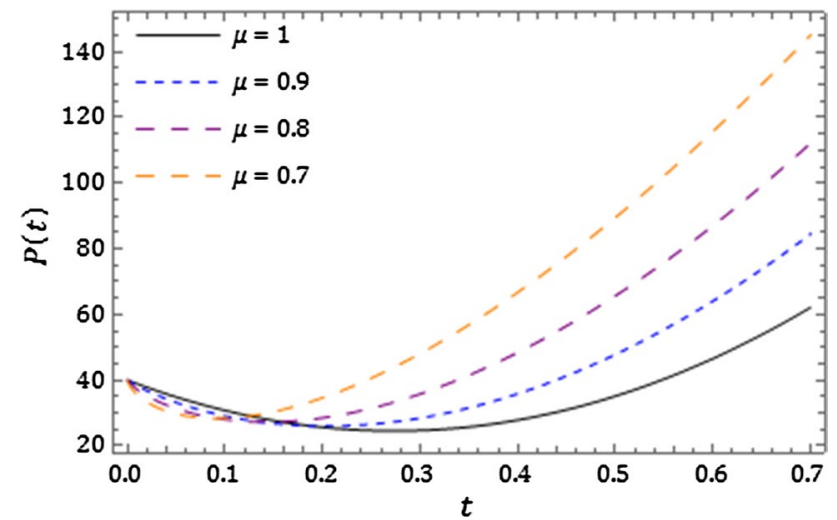

Fig. 1 Plots of $q$-HATM solution for potential smoker $P(t)$ with respect to $t$ at $\hbar=-1, n=1$ and using Table 1 for diverse $\mu$ 


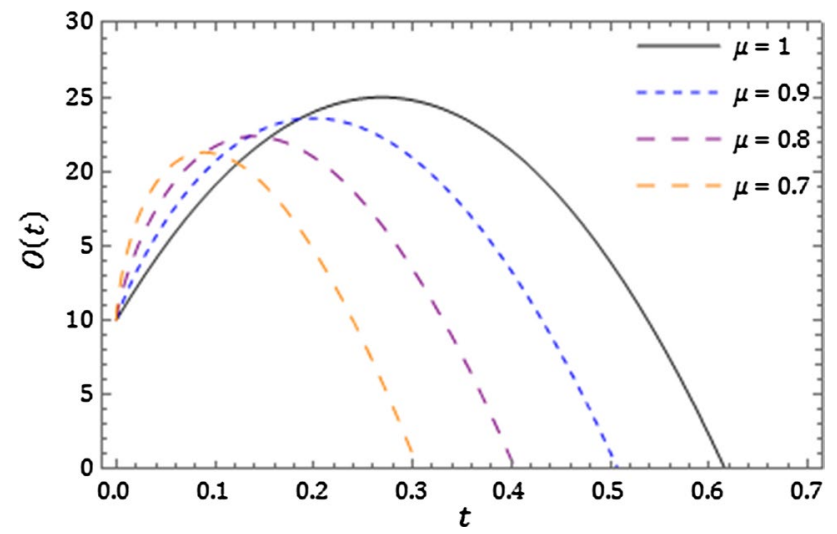

Fig. 2 Response of $q$-HATM solution for occasional smoker $O(t)$ with respect to $t$ at $\hbar=-1, n=1$ and using Table 1 for different $\mu$

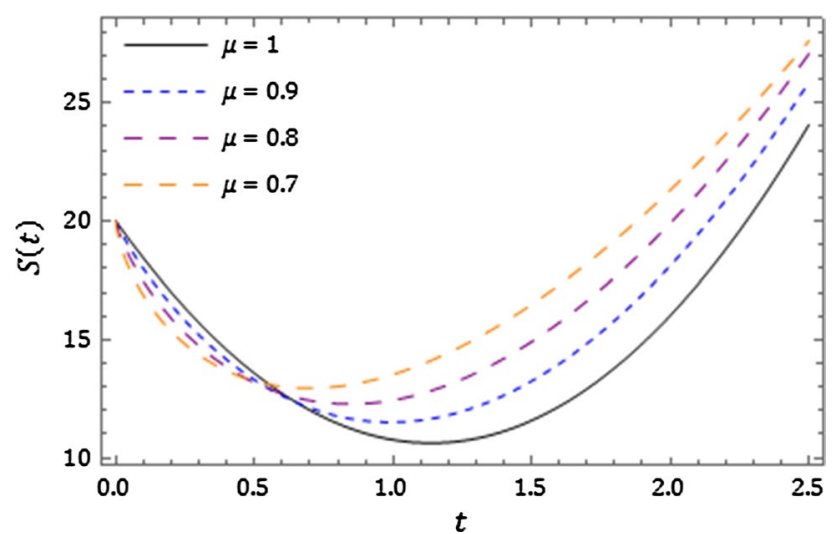

Fig. 3 Nature of $q$-HATM solution for smoker $S(t)$ with respect to $t$ at $\hbar=-1, n=1$ and using Table 1 for diverse $\mu$

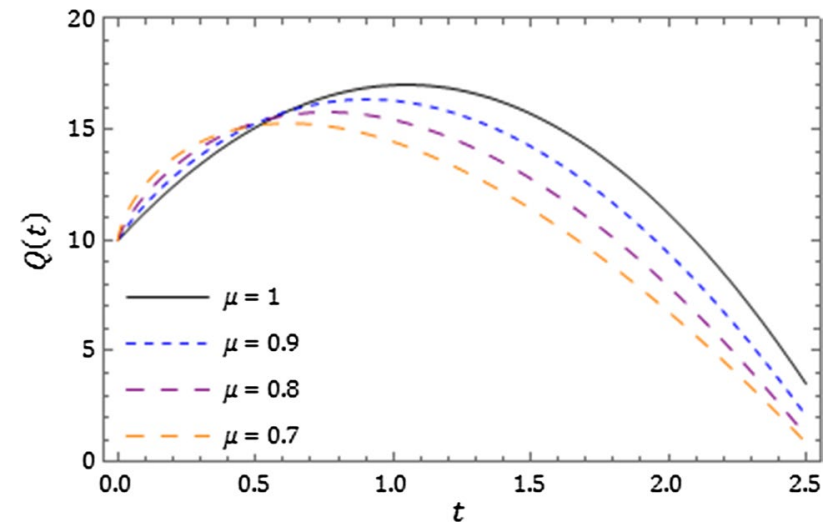

Fig. 4 Plot of $q$-HATM solution for temporarily quit smoker $Q(t)$ with respect to $t$ at $\hbar=-1, n=1$ and using Table 1 for different $\mu$

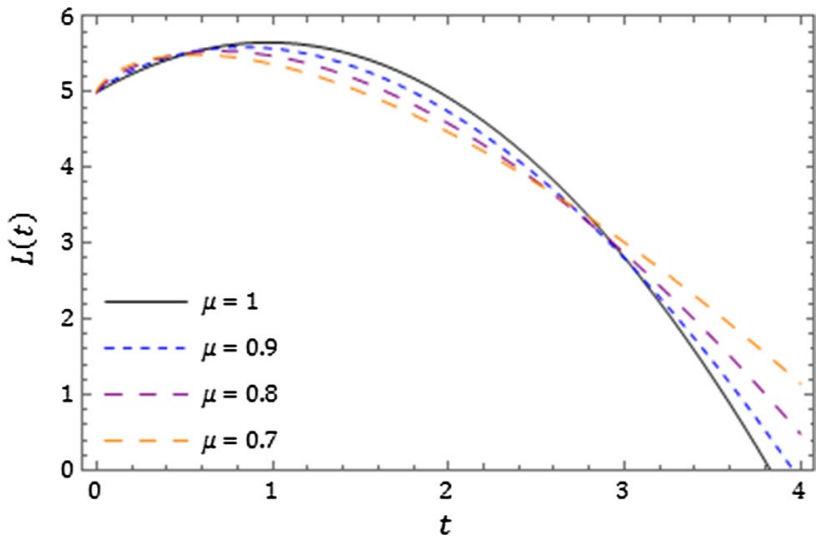

Fig. 5 Nature of $q$-HATM solution for permanently quit smoker $Q(t)$ with respect to $t$ at $\hbar=-1, n=1$ and using Table 1 for distinct $\mu$

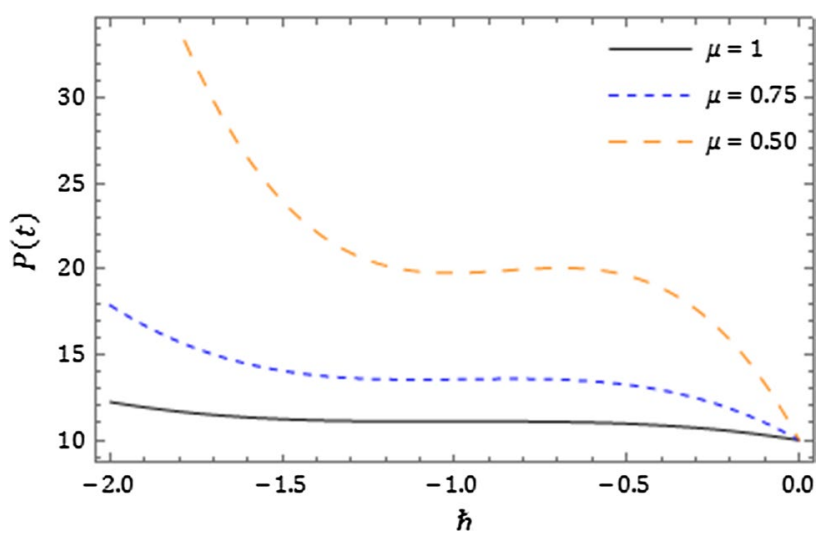

Fig. $6 \hbar$-curves drown for $P(t)$ with diverse $\mu$ at $t=0.01, n=1$ and using Table 1

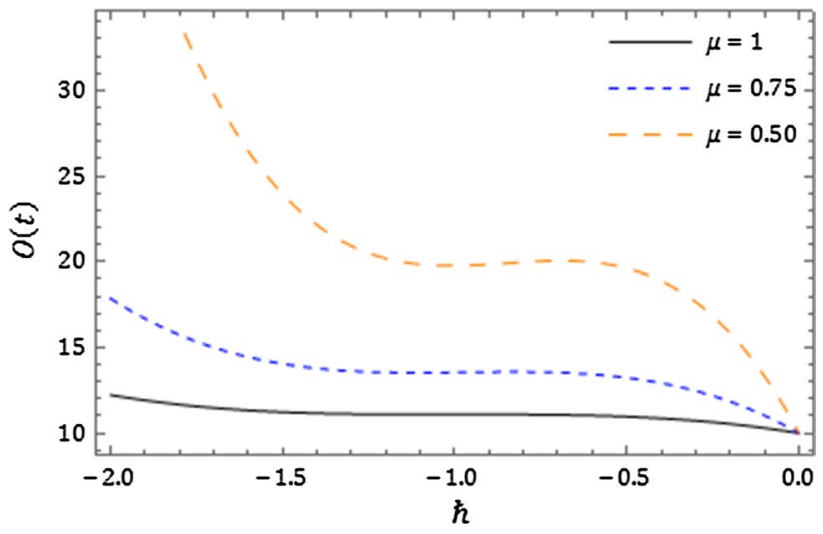

Fig. $7 \hbar$-curves drown for $O(t)$ with distinct $\mu$ at $t=0.01, n=1$ and using Table 1 


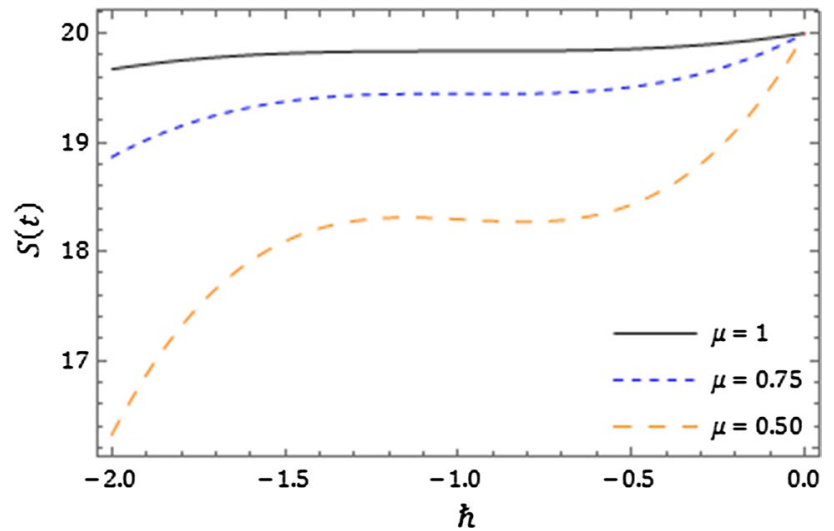

Fig. $8 \hbar$-curves drown for $S(t)$ with different $\mu$ at $t=0.01, n=1$ and using Table 1

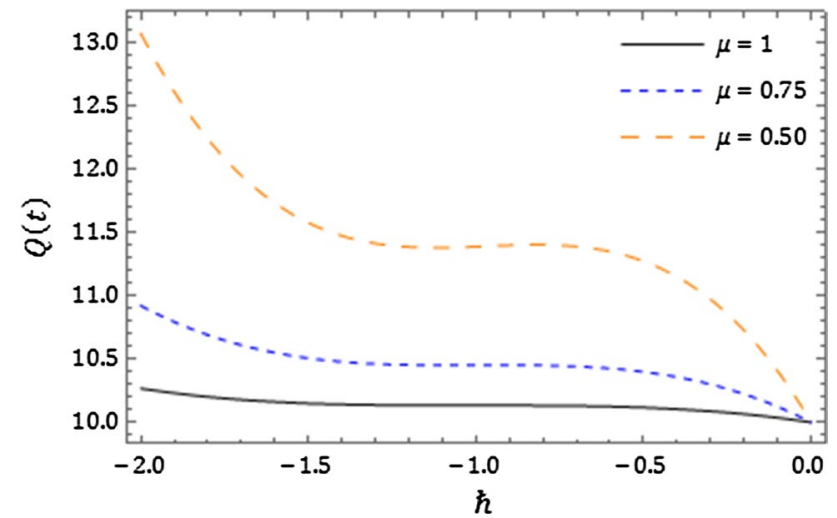

Fig. $9 \hbar$-curves plotted for $Q(t)$ with distinct $\mu$ at $t=0.01, n=1$ and using Table 1

$$
\begin{aligned}
O_{3}(t)= & \frac{(n+\hbar)^{2} \hbar\left(10\left(-80 \beta+\eta+\alpha_{1}\right)\right) t^{\mu}}{\Gamma[\mu+1]} \\
& +\frac{10(n+\hbar) \hbar^{2}\left(-1600 \beta^{2}-80 \beta \gamma-240 \beta \eta+\eta^{2}+2 \beta \lambda+(-40 \beta+2 \eta) \alpha_{1}+\alpha_{1}^{2}+800 \beta \alpha_{2}\right) t^{2 \mu}}{\Gamma[2 \mu+1]} \\
& +\frac{10 \hbar^{3} t^{3 \mu}}{\Gamma[\mu+1]^{2} \Gamma[3 \mu+1]}\left(\left(\beta(800 \beta+40 \eta-\lambda)\left(\alpha_{1}-2\left(\gamma+\eta-10 \alpha_{2}\right)\right)\right) \Gamma[2 \mu+1]\right. \\
& +\Gamma[\mu+1]^{2}\left(-32000 \beta^{3}+\eta^{3}-80 \beta\left(\gamma^{2}+3 \gamma \eta+5 \eta^{2}\right)-40 \beta^{2}(40(\gamma+4 \eta)-\lambda)\right. \\
& +2 \beta(1+2 \eta) \lambda+\alpha_{1}\left(-4000 \beta^{2}+3 \eta^{2}+2 \beta(-20(\gamma+5 \eta)+\lambda)+3 \eta \alpha_{1}+\alpha_{1}^{2}\right) \\
& \left.\left.+400 \beta\left(40 \beta+4 \gamma \delta+8 \eta+\alpha_{1}\right) \alpha_{2}+8000 \beta \alpha_{2}^{2}\right)\right), \\
& =\frac{(n+\hbar)^{2} \hbar\left(-10 \alpha_{1}+20\left(\gamma+\eta-10 \alpha_{2}\right)\right) t^{\mu}}{\Gamma[\mu+1]} \\
& -\frac{10(n+\hbar) \hbar^{2} t^{2 \mu}\left(\alpha_{1}^{2}+\alpha_{1}\left(-80 \beta+\gamma+2 \eta-10 \alpha_{2}\right)\right.}{\Gamma[2 \mu+1]} \\
& \left.-2\left((\gamma+\eta)^{2}-10(2 \gamma \delta+3 \eta) \alpha_{2}-100 \alpha_{2}^{2}\right)\right)-\frac{10 \hbar^{3} t^{3 \mu}}{\Gamma[\mu+1]^{2} \Gamma[3 \mu+1]}\left(\left(-2(\gamma+\eta)^{3}\right.\right. \\
& +20\left(\gamma^{2}(-1+4 \delta)+5 \eta^{2}+\gamma(\eta+6 \delta \eta)\right)+\alpha_{1}^{3}+\alpha_{1}^{2}\left(-40 \beta+\gamma+3 \eta-10 \alpha_{2}\right) \\
& +200(\gamma+4 \eta) \alpha_{2}^{2}+2000 \alpha_{2}^{3}-\alpha_{1}\left(1600 \beta^{2}-\gamma^{2}-3 \gamma \eta-3 \eta^{2}+2 \beta(80 \gamma+160 \eta-\lambda)\right. \\
& \left.\left.+(-1600 \beta+20 \gamma \delta+30 \eta) \alpha_{2}+100 \alpha_{2}^{2}\right)\right) \Gamma[\mu+1]^{2} \\
& \left.+10 \Gamma[2 \mu+1]\left(-\alpha_{1}+2\left(\gamma+\eta-10 \alpha_{2}\right)\right) \alpha_{2}\left(2 \gamma(-1+\delta)+\eta+20 \alpha_{2}\right)\right),
\end{aligned}
$$




$$
\begin{aligned}
Q_{3}(t)= & \frac{(n+\hbar)^{2} \hbar\left(10\left(2 \gamma(-1+\delta)+\eta+20 \alpha_{2}\right)\right) t^{\mu}}{\Gamma[\mu+1]} \\
& +\frac{10(n+\hbar) \hbar^{2} t^{2 \mu}}{\Gamma[2 \mu+1]}\left(-2 \gamma^{2}+2 \gamma^{2} \delta-4 \gamma \eta+4 \gamma \delta \eta+\eta^{2}\right. \\
& \left.+\alpha_{1}\left(\gamma-\gamma \delta-10 \alpha_{2}\right)+20(\gamma \delta+3 \eta) \alpha_{2}+200 \alpha_{2}^{2}\right)-\frac{10 \hbar^{3} t^{3 \mu}}{\Gamma[\mu+1]^{2} \Gamma[3 \mu+1]}\left(\left(-2 \gamma^{3}(-1+\delta)\right.\right. \\
& -2 \gamma^{2}(-1+\delta) \eta+2 \gamma(-1+\delta) \eta^{2}+\eta^{3}+\alpha_{1}^{2}\left(\gamma(-1+\delta)-10 \alpha_{2}\right)+20\left(\gamma^{2}\left(-1+2 \delta^{2}\right)\right. \\
& \left.+\gamma(-5+8 \delta) \eta+5 \eta^{2}\right) \alpha_{2}+200(\gamma(-1+\delta)+4 \eta) \alpha_{2}^{2}+2000 \alpha_{2}^{3} \\
& \left.-\alpha_{1}\left(-\gamma(-1+\delta)(-80 \beta+\gamma+\eta)-10(80 \beta+2 \gamma-3 \gamma \delta-3 \eta) \alpha_{2}+100 \alpha_{2}^{2}\right)\right) \Gamma[\mu+1]^{2} \\
& \left.+10 \Gamma[2 \mu+1]\left(-\alpha_{1}+2\left(\gamma+\eta-10 \alpha_{2}\right)\right) \alpha_{2}\left(2 \gamma(-1+\delta)+\eta+20 \alpha_{2}\right)\right), \\
L_{3}(t)= & \frac{(n+\hbar)^{2} \hbar(-20 \gamma \delta+5 \eta) t^{\mu}}{\Gamma[\mu+1]} \\
& +\frac{5(n+\hbar) \hbar^{2}\left(-4 \gamma^{2} \delta-8 \gamma \delta \eta+\eta^{2}+2 \gamma \delta \alpha_{1}+40 \gamma \delta \alpha_{2}\right) t^{2 \mu}}{\Gamma[2 \mu+1]} \\
& -\frac{5 \hbar^{3}\left(4 \gamma^{3} \delta+12 \gamma^{2} \delta \eta+12 \gamma \delta \eta^{2}-\eta^{3}-2 \gamma \delta \alpha_{1}^{2}-2 \gamma \delta \alpha_{1}\left(-80 \beta+\gamma+3 \eta-10 \alpha_{2}\right)-80 \gamma \delta(\gamma \delta+2 \eta) \alpha_{2}-400 \gamma \delta \alpha_{2}^{2}\right) t^{3 \mu}}{\Gamma[3 \mu+1]} \\
\vdots \quad &
\end{aligned}
$$

In this way, the rest of the iterative terms can be obtained. Then, the $\boldsymbol{q}$-HATM series solution for the system of Eq. (31) is given by

$$
\begin{aligned}
& P(t)=P_{0}(t)+\sum_{m=1}^{\infty} P_{m}(t)\left(\frac{1}{n}\right)^{m}, \\
& O(t)=O_{0}(t)+\sum_{m=1}^{\infty} O_{m}(t)\left(\frac{1}{n}\right)^{m}, \\
& S(t)=S_{0}(t)+\sum_{m=1}^{\infty} S_{m}(t)\left(\frac{1}{n}\right)^{m}, \\
& Q(t)=Q_{0}(t)+\sum_{m=1}^{\infty} Q_{m}(t)\left(\frac{1}{n}\right)^{m}, \\
& L(t)=L_{0}(t)+\sum_{m=1}^{\infty} L_{m}(t)\left(\frac{1}{n}\right)^{m} .
\end{aligned}
$$

\section{Numerical results and discussion}

In order to validate the proposed technique is reliable and efficient; the approximated analytical solutions have been evaluated. Tables 2, 3, 4, 5 and 6 show that the considered model is noticeably depends on time-fractional-order derivative. This guides us to understand the behaviour of smoking evolution. Figures 1, 2, 3, 4 and 5 explore the nature of obtained solutions for potential smoker $P(t)$, smoker $S(t)$, occasional smoker $O(t)$, permanently quit smoker $L(t)$ and temporarily quit smoker $Q(t)$ with change in time ( $t$ ) for distinct Brownian motion and standard motion $(\mu=1)$, and from these we see that the arbitrary-order smoking model has more degree of flexibility. Additionally, from these plots, we absorbed that the consequence of considered model is evidently depends on its history and the parameters mentioned in the system of Eq. (9). The $\hbar$-curves for the system considered in Eq. (31) at distinct values of $\mu$ are presented in Figs. 6, 7, 8, 9 and 10. For appropriate value of $\hbar$, series solutions rapidly converge to the exact solution in the cited scheme. Moreover, this helps us to control and adjust the region of convergence for the obtained series solution. From

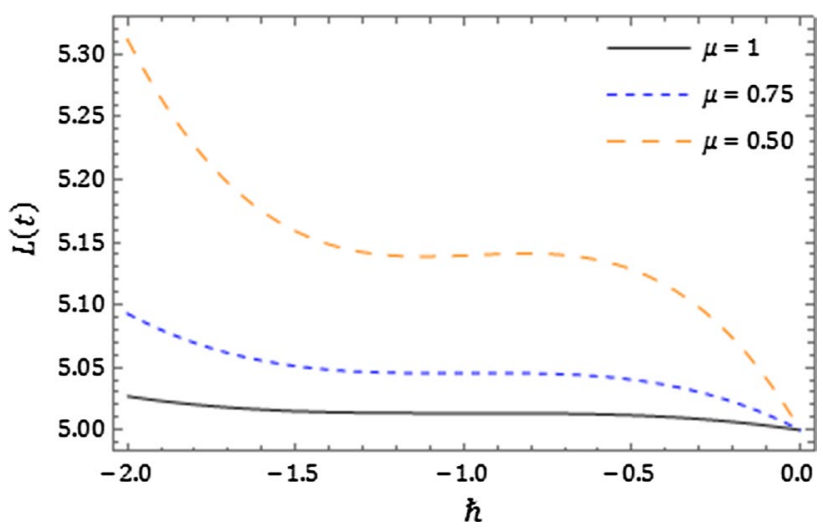

Fig. $10 \hbar$-curves drown for $L(t)$ with diverse $\mu$ at $t=0.01, n=1$ and using Table 1 
the obtained results, we can see that the proposed scheme is helpful to understand the behaviour with the aid of fractional derivative.

\section{Conclusion}

In present framework, the approximated analytical solutions for the smoking epidemic model through $q$-homotopy analysis transform method were investigated. The fractional equations involved in the considered model are of the Caputo sense. The obtained solutions are given in a series form, which converges rapidly. The graphical representation shows that the model depends notably on the fractional order and the selected system of parameters, and these parameters can influence the stability of the model. The dynamic behaviour of smoking model depends on both the time instant and the time history, which can be efficiently modelled using the
Proof For Eq. (13), the solution is defined by

$v(x, t)=\sum_{m=0}^{\infty} v_{m}(x, t)$,

where

$$
\begin{aligned}
v_{m}(x, t)= & \left(\mathrm{k}_{m}+\hbar\right) v_{m-1}(x, t)-\left(1-\frac{\mathrm{k}_{m}}{n}\right) L^{-1}\left(\sum_{k=0}^{n-1} s^{\mu-k-1} v^{(k)}(x, 0)\right. \\
& \left.+\frac{1}{s^{\mu}} L[f(x, t)]\right)+\hbar L^{-1}\left[\frac{1}{s^{\mu}} L\left[R v_{m-1}+\mathcal{H}_{m-1}\right]\right] .
\end{aligned}
$$

If possible, let $v$ and $v^{\star}$ be two distinct solutions of Eq. (13); then, with the help of the above equation, we obtain

$\left|v-v^{\star}\right|=\left|(n+\hbar)\left(v-v^{\star}\right)+\hbar L^{-1}\left(\frac{1}{s^{\alpha}} L\left(N\left(v-v^{\star}\right)+R\left(v-v^{\star}\right)\right)\right)\right|$.

Then, by using the convolution theorem for $L T$, we have

$$
\begin{aligned}
\left|v-v^{\star}\right| & \leq(n+\hbar)\left|v-v^{\star}\right|+\hbar \int_{0}^{t}\left(\left|N\left(v-v^{\star}\right)\right|+\left|R\left(v-v^{\star}\right)\right|\right) \frac{(t-\xi)^{\mu}}{\Gamma(\mu+1)} \mathrm{d} \xi \\
& \leq(n+\hbar)\left|v-v^{\star}\right|+\hbar \int_{0}^{t}\left(\epsilon\left|\left(v-v^{\star}\right)\right|+\mu\left|\left(v-v^{\star}\right)\right|\right) \frac{(t-\xi)^{\mu}}{\Gamma(\mu+1)} \mathrm{d} \xi .
\end{aligned}
$$

theory of fractional calculus. By considering the Caputo fractional derivatives, we realize that we have stabilized a more competent realistic model. Finally, we conclude that the proposed technique is highly methodical as well as more effective and it can be used to examine nonlinear fractional mathematical models describing biological phenomena, and the use of fractional calculus opens the new paradigms in the area of mathematical modelling.

Open Access This article is distributed under the terms of the Creative Commons Attribution 4.0 International License (http://creativeco mmons.org/licenses/by/4.0/), which permits unrestricted use, distribution, and reproduction in any medium, provided you give appropriate credit to the original author(s) and the source, provide a link to the Creative Commons license, and indicate if changes were made.

\section{Appendix}

Here, we present proof for the Theorems 2 and 3.

Theorem 2 (Uniqueness theorem) [38] The solution for the nonlinear fractional differential Eq. (13) obtained by $q$-HATM is unique for every $\beta \in(0,1)$, where $\beta=(n+\hbar)+\hbar(\epsilon+\mu) T$.
By the aid of integral mean value theorem, the above equation reduces to

$\left|v-v^{\star}\right| \leq(n+\hbar)\left|v-v^{\star}\right|+\hbar\left(\epsilon\left|\left(v-v^{\star}\right)\right|+\mu\left|\left(v-v^{\star}\right)\right|\right) T$.

Here, $\beta=(n+\hbar)+\hbar(\epsilon+\mu) T$; thus,

$\left|v-v^{\star}\right| \leq \beta\left|v-v^{\star}\right| \Rightarrow(1-\beta)\left|v-v^{\star}\right| \leq 0$

Since $0<\beta<1$, then $v-v^{\star}=0 \Rightarrow v=v^{\star}$. Hence, the solution for Eq. (5) is unique.

Theorem 3 (Convergence theorem) [38] Let X be a Banach space and $F: X \rightarrow X$ be a nonlinear mapping. Assume that $\|F(v)-F(w)\| \leq \beta\|v-w\|, \quad \forall a, b \in X$,

then $F$ has a fixed point in view of Banach fixed point theory. Moreover, for the arbitrary selection of $a_{0}, b_{0} \in X$, the sequence generated by the q-HATM converges to fixed point of $F$ and

$v_{m}-v_{n} \leq \frac{\beta^{n}}{1-\beta} v_{1}-v_{0}, \quad \forall a, b \in X$ 
Proof For all continuous functions, let us consider Banach space $(C[I],\|\cdot\|)$ on $I$ with norm is given by $\|g(\lambda)\|=\max _{\lambda \in \mathrm{I}}|g(\lambda)|$. First, we prove that $\left\{v_{n}\right\}$ is Cauchy sequence in $X$.

Now consider,
But $\left\|v_{1}-v_{0}\right\|<\infty$, consequently as $m \rightarrow \infty$, then $\left\|v_{m}-v_{n}\right\| \rightarrow 0$.

It provides $\left\{v_{n}\right\}$ is Cauchy sequence in $C[I]$, and every Cauchy sequence is convergent sequence. Hence, $\left\{v_{n}\right\}$ is convergent sequence.

$$
\begin{aligned}
\left\|v_{m}-v_{n}\right\| & =\max _{\lambda \in I}\left|v_{m}-v_{n}\right| \\
& =\max _{\lambda \in I}\left|(n+\hbar)\left(v_{m-1}-v_{n-1}\right)+\hbar L^{-1}\left(\frac{1}{s^{\alpha}} L\left(N\left(v_{m-1}-v_{n-1}\right)+R\left(v_{m-1}-v_{n-1}\right)\right)\right)\right| \\
& \leq \max _{\lambda \in I}\left[(n+\hbar)\left|\left(v_{m-1}-v_{n-1}\right)\right|+\hbar L^{-1}\left(\frac{1}{s^{\alpha}} L\left(N\left|v_{m-1}-v_{n-1}\right|+R\left(\left|v_{\mathrm{m}-1}-v_{\mathrm{n}-1}\right|\right)\right)\right) .\right.
\end{aligned}
$$

By the convolution theorem for Laplace transform, Eq. (40) becomes

$$
\begin{aligned}
\left\|v_{m}-v_{n}\right\| \leq & \max _{\lambda \in I}\left[(n+\hbar)\left|\left(v_{m-1}-v_{n-1}\right)\right|\right. \\
& +\hbar \int_{0}^{t}\left(\left|N\left(v_{m-1}-v_{n-1}\right)\right|+\left|R\left(v_{m-1}-v_{n-1}\right)\right|\right) \frac{(t-\xi)^{\alpha}}{\Gamma(\alpha+1)} \mathrm{d} \xi \\
\leq & \max _{\lambda \in I}\left[(n+\hbar)\left|\left(v_{m-1}-v_{n-1}\right)\right|\right. \\
& +\hbar \int_{0}^{t}\left(\left|N\left(v_{m-1}-v_{n-1}\right)\right|+\left|R\left(v_{m-1}-v_{n-1}\right)\right|\right) \frac{(t-\xi)^{\alpha}}{\Gamma(\alpha+1)} \mathrm{d} \xi .
\end{aligned}
$$

By the aid of integral mean value theorem, the above inequality reduces to

$$
\begin{aligned}
\left\|v_{m}-v_{n}\right\| \leq & \max _{\lambda \in I}\left[(n+\hbar)\left|\left(v_{m-1}-v_{n-1}\right)\right|\right. \\
& \left.+\hbar\left(\epsilon\left|v_{m-1}-v_{n-1}\right|+\mu\left|v_{m-1}-v_{n-1}\right|\right) T\right] \\
\leq & \beta|| v_{m-1}-v_{n-1}|| .
\end{aligned}
$$

For $m=n+1$, one can get

$$
\begin{aligned}
\left\|v_{n+1}-v_{n}\right\| & \leq\left\|\beta v_{n}-v_{n-1}\right\| \leq \beta^{2}\left\|v_{n-1}-v_{n-2}\right\| \\
& \leq \beta^{3}\left\|v_{n-2}-v_{n-3}\right\| \leq \cdots \leq \beta^{n}\left\|v_{1}-v_{0}\right\| .
\end{aligned}
$$

In view of triangular inequality, we have

$$
\begin{aligned}
\left\|v_{m}-v_{n}\right\| & \leq\left\|v_{n+1}-v_{n}\right\|+\left\|v_{n+2}-v_{n+1}\right\|+\cdots+\left\|v_{m}-v_{m-1}\right\| \\
& \leq\left[\beta^{n}+\beta^{n+1}+\cdots+\beta^{m-1}\right]\left\|v_{1}-v_{0}\right\| \\
& =\beta^{n}\left[1+\beta+\cdots+\beta^{m-n-1}\right]\left\|v_{1}-v_{0}\right\| \\
& \leq \beta^{n}\left[\frac{1-\beta^{m-n-1}}{1-\beta}\right]\left\|v_{1}-v_{0}\right\| .
\end{aligned}
$$

Clearly, $1-\beta^{m-n-1}<1($ since $0<\beta<1)$. Therefore, the above inequality becomes

$\left\|v_{m}-v_{n}\right\| \leq \frac{\beta^{n}}{1-\beta}\left\|v_{1}-v_{0}\right\|$.

\section{References}

1. Bernoulli, D.: Essai d'une nouvelle analyse de la mortalite causee par la petite verole. Mem. Math. Phys. Acad. R Sci. 1-41 (1766)

2. Ross, R., Annett, H.E., Austen, E.E.: Report of the Malaria Expedition of the Liverpool School of Tropical Medicine and Medical Parasitology, pp. 1-71. University Press of Liverpool, Liverpool (1900). https://doi.org/10.5962/bhl.title.99657

3. Santonja, F.J., Sanchez, E., Rubio, M., Morera, J.L.: Alcohol consumption in Spain and its economic costs: a mathematical modeling approach. Math. Comput. Model. 52, 999-1003 (2010)

4. Santonja, F.J., Villanueva, R.J., Jodar, L., Gonzalez, G.: Mathematical modeling of social obesity epidemic in the region of Valencia, Spain. Math. Comput. Model. Dyn. Syst. 16(1), 23-34 (2010)

5. Sanchez, E., Villanueva, R.J., Santonja, F.J., Rubio, M.: Predicting cocaine consumption in Spain. A mathematical modeling approach. Drugs Educ. Prev. Policy 18(2), 105-108 (2011)

6. Guerrero, F., Santonja, F.J., Villanueva, R.J.: Analysing the effect of Spanish smoke-free legislation of year 2006: a new method to quantify its impact using a dynamic model. Int. J. Drug Policy 22, 247-251 (2011)

7. WHO, 2010. http://www.emro.who.int/tfi/facts.htm\#fact2

8. Castillo-Garsow, C., Jordan-Salivia, G., Herrera, A.R.: Mathematical models for the dynamics of tobacco use, recovery, and relapse. In: Technical Report Series BU-1505-M, Cornell University, Ithaca, NY, USA (1997)

9. Huo, H.F., Zhu, C.C.: Influence of relapse in a giving up smoking model. Abstr. Appl. Anal. (2013). https://doi. org/10.1155/2013/525461

10. Lahrouz, A., Omari, L., Kiouach, D., Belmaati, A.: Deterministic and stochastic stability model of smoking. Stat. Probab. Lett. 81(8), 1276-1284 (2011)

11. Erturk, V.S., Zaman, G., Momani, S.: A numeric analytic method for approximating a giving up smoking model containing fractional derivatives. Comput. Math Appl. 64, 3068-3074 (2012)

12. Guerrero, F., Santonja, F.J., Villanueva, R.J.: Solving a model for the evolution of smoking habit in Spain with homotopy analysis method. Nonlinear Anal. Real World Appl. 14, 549-558 (2013)

13. Van Voorn, G.A.K., Kooi, B.W.: Smoking epidemic eradication in a eco-epidemiological dynamical model. Ecol. Complex 14, 180-189 (2013)

14. Singh, J., Kumar, D., Qurashi, M.A., Baleanu, D.: A new fractional model for giving up smoking dynamics. Adv. Differ. Equ. 88, 1-16 (2017). https://doi.org/10.1186/s13662-017-1139-9

15. Sikander, W., Khan, U., Ahmed, N., Mohyud-Din, S.T.: Optimal solutions for a bio mathematical model for the evolution of smoking habit. Results Phys. 7, 510-517 (2017) 
16. Mondol, A., Gupta, R., Das, S., Dutta, T.: An insight into Newton's cooling law using fractional calculus. J. Appl. Phys. 123(6), 3-10 (2018)

17. Azarang, A., Ghassemian, H.: Application of fractional-order differentiation in multispectral image fusion. Remote Sens. Lett. 9(1), 91-100 (2018)

18. Esen, A., Sulaiman, T.A., Bulut, H., Baskonus, H.M.: Optical solitons to the space-time fractional $(1+1)$-dimensional coupled nonlinear Schrodinger equation. Optik 167, 150-156 (2018)

19. Baskonus, H.M., Hammouch, Z., Mekkaoui, T., Bulut, H.: Chaos in the fractional order logistic delay system: circuit realization and synchronization. AIP Conf. Proc. 1738, 290005 (2016)

20. Bulut, H., Kumar, D., Singh, J., Swroop, R., Baskonus, H.M.: Analytic study for a fractional model of HIV infection of CD4+ T lymphocyte cells. Math. Nat. Sci. 2(1), 33-43 (2018)

21. Dokuyucu, M.A., Celik, E., Bulut, H., Baskonus, H.M.: Cancer treatment model with the Caputo-Fabrizio fractional derivative. Eur. Phys. J. Plus. 133(92), 1-7 (2018)

22. Haq, F., Shah, K., Khan, A., Shahzad, M., Rahman, G.: Numerical solution of fractional order epidemic model of a vector born disease by Laplace Adomian decomposition method. Punjab Univ. J. Math. 49(2), 13-22 (2017)

23. Liao, S.J.: Homotopy analysis method and its applications in mathematics. J Basic Sci Eng 5(2), 111-125 (1997)

24. Liao, S.J.: Homotopy analysis method: a new analytic method for nonlinear problems. Appl. Math. Mech. 19, 957-962 (1998)

25. Singh, J., Kumar, D., Swroop, R.: Numerical solution of timeand space-fractional coupled Burgers' equations via homotopy algorithm. Alexandria Eng. J. 55(2), 1753-1763 (2016)

26. Veeresha, P., Prakasha, D.G., Baskonus, H.M.: New numerical surfaces to the mathematical model of cancer chemotherapy effect in Caputo fractional derivatives. Chaos 29, 013119 (2019). https ://doi.org/10.1063/1.5074099

27. Srivastava, H.M., Kumar, D., Singh, J.: An efficient analytical technique for fractional model of vibration equation. Appl. Math. Model. 45, 192-204 (2017)

28. Veeresha, P., Prakasha, D.G., Baskonus, H.M.: Novel simulations to the time-fractional Fisher's equation. Math. Sci. (2019). https ://doi.org/10.1007/s40096-019-0276-6
29. Prakasha, D.G., Veeresha, P., Rawashdeh, M.S.: Numerical solution for $(2+1)$-dimensional time-fractional coupled Burger equations using fractional natural decomposition method. Math. Meth. Appl. Sci. (2019). https://doi.org/10.1002/mma.5533

30. Liouville, J.: Memoire sur quelques questions de geometrie et de mecanique, et sur un nouveau genre de calcul pour resoudre ces questions. J. Ecole Polytech. 13, 1-69 (1832)

31. Riemann, G.F.B.: Versuch einer allgemeinen Auffassung der Integration und Differentiation. Gesammelte Mathematische Werke, Leipzig (1896)

32. Caputo, M.: Elasticita e Dissipazione. Zanichelli, Bologna (1969)

33. Podlubny, I.: Fractional Differential Equations. Academic, New York (1999)

34. Abdullah, M., Ahmad, A., Raza, N., Farman, M., Ahmad, M.O.: Approximate solution and analysis of smoking epidemic model with Caputo fractional derivatives. Int. J. Appl. Comput. Math. 4(112), 1-16 (2018). https://doi.org/10.1007/s40819-018-0543-5

35. Ullah, R., Khan, M., Zaman, G.: Dynamical features of a mathematical model on smoking. J. Appl. Environ. Biol. Sci. 6(1), 92-96 (2016)

36. Prakash, A., Veeresha, P., Prakasha, D.G., Goyal, M.: A homotopy technique for fractional order multi-dimensional telegraph equation via Laplace transform. Eur. Phys. J. Plus 134(19), 1-18 (2019). https://doi.org/10.1140/epjp/i2019-12411-y

37. Veeresha, P., Prakasha, D.G., Magesh, N., Nandeppanavar, M.M., Christopher, A.J.: Numerical simulation for fractional JaulentMiodek equation associated with energy-dependent Schrodinger potential using two novel techniques. (2019). arXiv:1810.06311 [math.NA]

38. Kumara, D., Singha, J., Baleanu, D.: A new analysis for fractional model of regularized long-wave equation arising in ion acoustic plasma waves. Math. Methods Appl. Sci. 40, 5642-5653 (2017)

39. Magrenan, A.A.: A new tool to study real dynamics: the convergence plane. Appl. Math. Comput. 248, 215-224 (2014)

Publisher's Note Springer Nature remains neutral with regard to jurisdictional claims in published maps and institutional affiliations. 